\title{
Bone augmentation in atrophic maxillary ridge by ridge split technique and guided bone regeneration: A case report
}

\author{
Romesh Soni ${ }^{1 *}$, Aditi Priya ${ }^{2}$, Himanshi Yadav ${ }^{3}$, Vikram Kumar ${ }^{4}$ \\ ${ }^{1}$ Professor, ${ }^{2-4}$ Post Graduate Student, Dept. of Prosthodontics, ${ }^{1-4}$ Institute of Medical Sciences, Banaras Hindu University, Varanasi, Uttar \\ Pradesh, India
}

\begin{abstract}
Bone loss after tooth extraction is inevitable, it can be horizontal, vertical bone loss or combination. Now a days, dental implants are emerging as a better treatment option for oral rehabilitation and become an integral part of dentistry ,thus availability of adequate bone volume in all 3-dimensions to stabilize implant is foremost requirement for successful treatment. Various methods have been used to augment the ridge bone volume which includes bone grafting along with guided bone regeneration, ridge split technique, sinus lift procedure and distraction osteogenesis. This case report presents aesthetic rehabilitation by implant retained prosthesis in upper anterior region with large defect by combining bone augmentation with ridge split technique to enable implant placement.
\end{abstract}

Keywords: Dental implants, Ridge split technique, Bone augmentation, Bone grafting, and Implant retained prosthesis.

\section{Introduction}

Tooth loss associated with trauma usually leads to progressive bone volume resorption resulting into thin atrophic narrow ridges. The narrow ridge necessitates calls for additional procedures to augment bone volume so that implant can be placed to receive prosthesis. Management of such defects in aesthetic zone further complicates the situation. Implant placement is prosthetic driven not bone driven, so presence of sufficient bone volume is the foremost requisite for implant placement. ${ }^{1}$ Ridge augmentation using auto graft and onlay block graft has resulted into successful outcomes, but the disadvantages are additional donar site and delayed implant placement for graft maturity. Therefore bone splitting and bone expansion can be carried out without much surgical trauma to patient. ${ }^{2}$

When bucco-palatal width is $3 \mathrm{~mm}$ to $6 \mathrm{~mm}$, ridge splitting with bone expansion is a viable option to augment bone for implant placement. The presence of trabeculae bone in between the cortices is foremost requisite to carry out ridge spit procedure. ${ }^{3}$

\section{Case Report}

A 25 year old male patient reported to Prosthodontics OPD, FDS, BHU with chief complaint of unpleasant appearance due to missing right upper front teeth due to trauma. On clinical examination, there were missing lateral incisor, canine and premolar teeth (Fig. 1). First treatment option advised to patient was removable partial denture but patient refused removable prosthesis as he was seeking for fixed prosthesis. Fixed prosthesis treatment options included fixed partial denture w.r.t.22, 23 and 24 but patient refused as he was not ready to sacrifice his healthy sound teeth as abutment for tooth preparation.Therefore the treatment plan decided for patient was implant retained prosthesis. The medical and dental history was non-contributory. The bucco-palatal width was deficit on palpation which was confirmed radiographically by OPG and Denta-scan (Fig. 2).

The patient was prepared for implant surgery after clearing routine blood investigations and a duly written consent form as a part of preparation protocol. Antibiotic prophylaxis was given before surgery. Lignocaine as local anesthesia with vasoconstrictor $(1: 80,000)$ was administered and mid-crestal incision was done with full thickness mucoperiosteum was reflected to expose surgical site. Clinically bucco-palatal width was narrower i.e. $2.5 \mathrm{~mm}$ to $3 \mathrm{~mm}$, Ridge split was planned to expand bone. Mid crestal cut was placed on bone using No.15 Bard Parker blade (Fig. 4) which was further extended deep to the length of implant, length was already determined by CBCT. Next, two vertical cut were placed, one each on both the ends of the horizontal cut to permit mobilization of cortical plate. Then, further splitting and expansion of bone was done by splitters and expand progressively. Subsequently ostetomies were done at slow speed of drill so that it expanded rather than cutting, this method was used to expand bucco-palatal width of bone so that bone was sufficient for implant diameter. After achieving sufficient bone expansion, three implants (each implant size- 3.5X13, TauregTM- S Adin dental implant systm LTD, Israel) were placed (Fig. 5). Bone graft (Geistlich Bio-Oss $0.25-1 \mathrm{~mm}$ particle size, $0.5 \mathrm{gm}, 1 \mathrm{cc}$, Germany) was placed at deficient site and covered with resorbable collagen membrane(perio-colRGTR/ HelisorbR-GTR sterile collagen membrane, Chennai, India) as barrier membrane (Fig. 6). As per standard protocol, flap scoring was done to attain flap approximation

\footnotetext{
*Corresponding Author: Romesh Soni, Dept. of Prosthodontics, Institute of Medical Sciences, Banaras Hindu University, Varanasi, Uttar Pradesh, India
}

Email: bhuprostho2019@gmail.com

http://doi.org/10.18231/j.aprd.2019.021 
and primary closure was attained using 3-0 silk suture. Postop medication prescribed for 7 days along with oral hygiene instructions.

Suture removal was done after 7 days. After 6 months surgical re-entry performed and provisional prosthesis was placed to attain desired gingival emergence profile (Fig. 7). After 1 month abutment preparation (Fig. 8) was done followed by open tray impression making and thus definite PFM (porcelain fused metal) prosthesis (Fig. 9) installed after occlusal adjustments.

\section{Results}

Insufficient bone volume can be augmented successfully by combining various methods of bone augmentation. This case resulted into successful bone augmentation by combining ridge split technique along with bone grafting supported by guided bone regeneration as collagen membrane have been used as barrier membrane. The overall result is successful PFM prosthetic rehabilitation in aesthetic area after bone augmentation procedure.

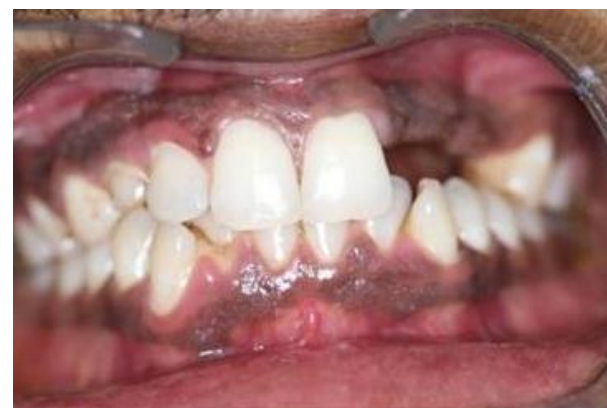

Fig. 1: Pre-op intraoral view

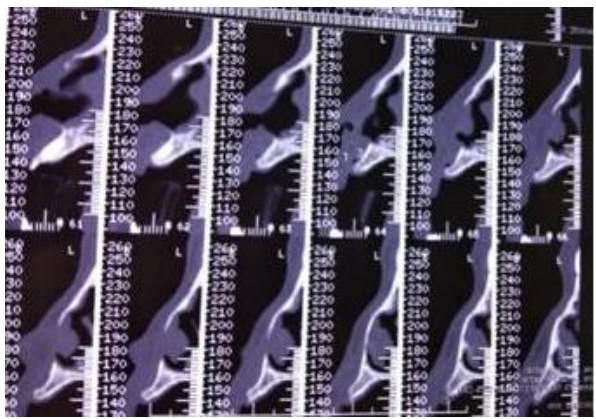

Fig. 2: Pre -op Radiograph

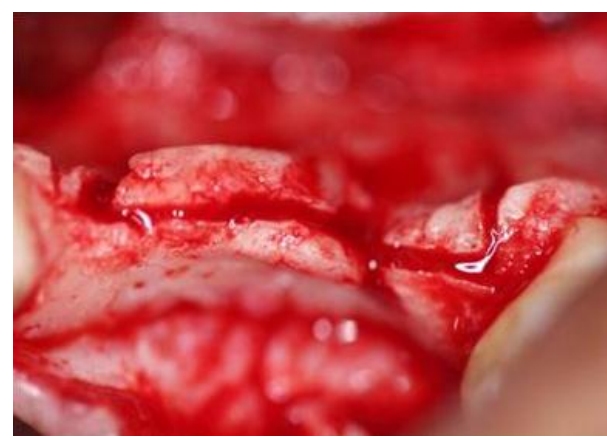

Fig. 3: Alveolar ridge showing Ridge split

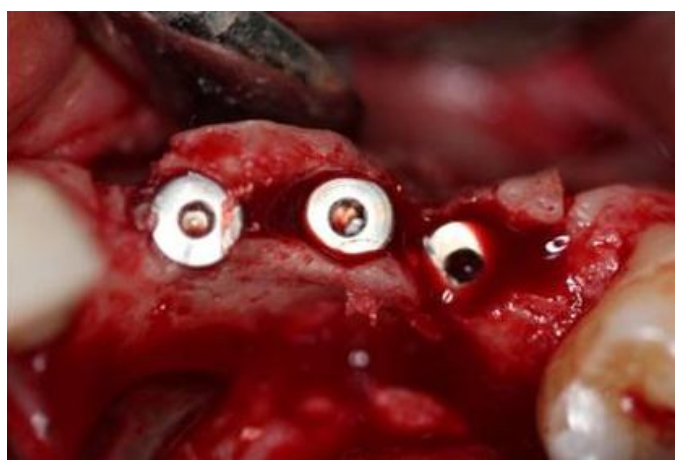

Fig. 4: Implant placement

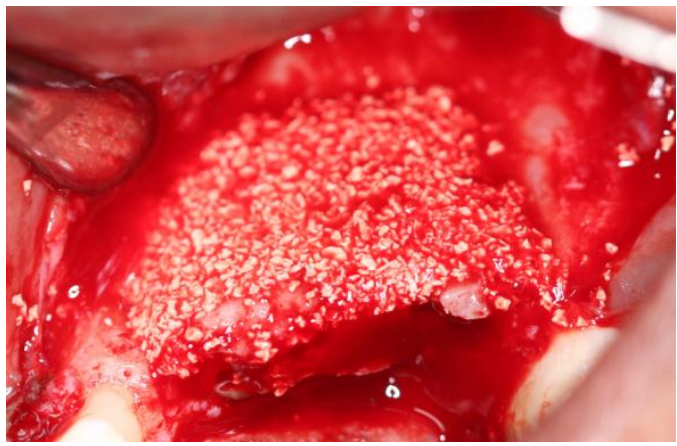

Fig. 5: Bone graft filled between implant and cortical plate

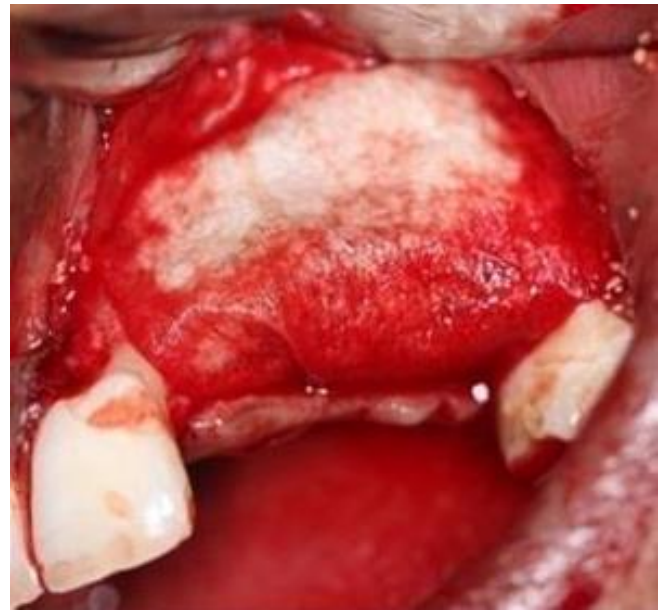

Fig. 6: Barrier membrane

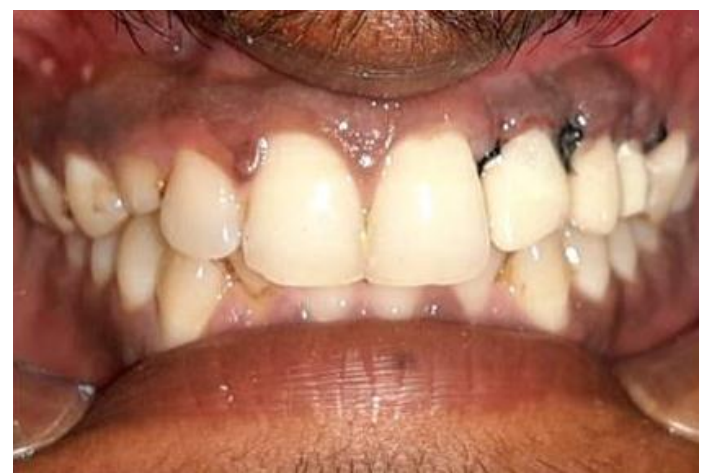

Fig. 7: Provisional restoration 


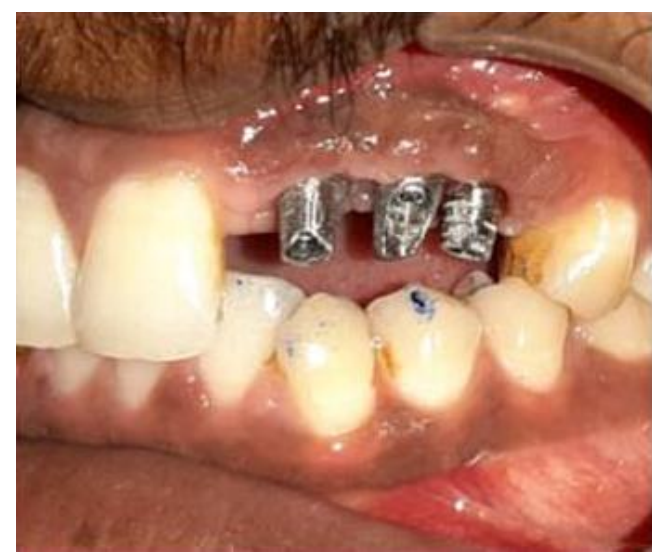

Fig. 8: Abutment preparation

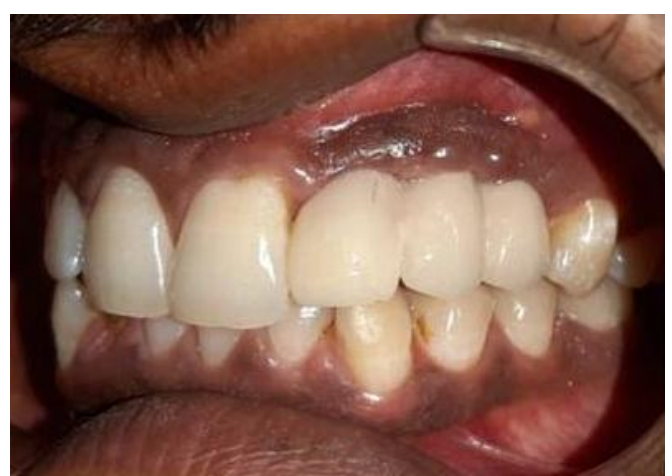

Fig. 9: Definite prosthesis

\section{Discussion}

Alveolar ridge split technique, a bone expansion technique was advocated by Dr Hilt tatum ${ }^{4}$. The advantage of ridge split is placement of implant in single surgery unlike guided tissue regeneration.

There are various instruments used in splitting of the ridge such as chisel, osteotome, disc bur, scalpel and mallet. A horizontal Incision is placed at the crest of the ridge along the mesio-distal direction. The depth of incision is extended upto nearly full length of the predetermined implant length and then two vertical incisions, one on both the ends of the horizontalal incision, are given to expand the ridges buccopalatally. According to Scipioni et al(2001), average ridge width increases from $2.4 \mathrm{~mm}$ to $6.0 \mathrm{~mm}$ using ridge split technique ${ }^{5}$. Maxilla is more suitable for ridge split technique than the mandible. ${ }^{6}$ Ideal indications for ridge split technique are those ridges that do not require vertical augmentation and have cancellous bone in between the buccal and lingual plates with a minimum buccolingual width of $4.0 \mathrm{~mm} .^{7}$ The disadvantage of this technique includes inability to increase the vertical height of the bone, fracture of the buccal and lingual plates, cannot be used when buccal defect is present, also there is a compromise in primary stability when the split is over extended. Sethi and Kaus performed a 5 year study with staged implants placed through maxillary expansion with osteotome and reported $97 \%$ success rate. ${ }^{8}$

\section{Conclusion}

This case report concludes that a patient with horizontal bone defect can be successfully rehabilitated by implant retained prosthesis when sufficient bone volume is achieved by involving ridge split technique along with bone grafting further supported by guided bone regeneration with collagen membrane as barrier membrane.

\section{Source of Funding}

None.

\section{Conflict of Interest}

None.

\section{References}

1. Scipionic A.,BruschiG.B.,calesini G.T edentulous ridge expansion technique:a five year study. Int $j$ Periodontics Restor Dent 1994;14:451-9

2. Soni R, Priya A, Yadav H, Mishra N, Kumar L . bone augmentation with sticky bone and platlet-rich fibrin by ridge split technique and nasal floor engagement for immediate loading of dental implant after extracting impacted crown. Natl J Maxillofac Surg 2019;10:98-101

3. Simion M, Baldoni M., Zaffe D. Jaw bone enlargement using immediate implant placement associated with split crest technique and guided tissue regeneration. Int J Periodontics Restor Dent 1992;12:462-73

4. Tatum H., Jr Maxillary and sinus implant reconstructions. Dent Clin North Am 1986;30:207-29.

5. Scipioni A, Bruschi GB, Giargia M, Berglundh T, Lindhe J. Healing at implants with and without primary bone contact. An experimental study in dogs. Clin Oral Implants Res 1997;8:3947.

6. Summers RB. A new concept in maxillary implant surgery: The osteotome technique. Compend Contin Educ Dent 1994;15:152-60.

7. Coatoam GW, Mariotti A. The segmental ridge split procedure. J Periodontal 2003;74:757-70

8. Sethi A, Kaus T. Maxillary ridge expansion with simultaneous implant placement: 5-year results of an ongoing clinical study. Int J Oral Maxillofac Implants 2000;15:491-9.

How to cite this article: Soni R, Priya A, Yadav H, Kumar V. Bone augmentation in atrophic maxillary ridge by ridge split technique and guided bone regeneration: A case report. Ann Prosthodont Restor Dent 2019;5(3):89-91. 